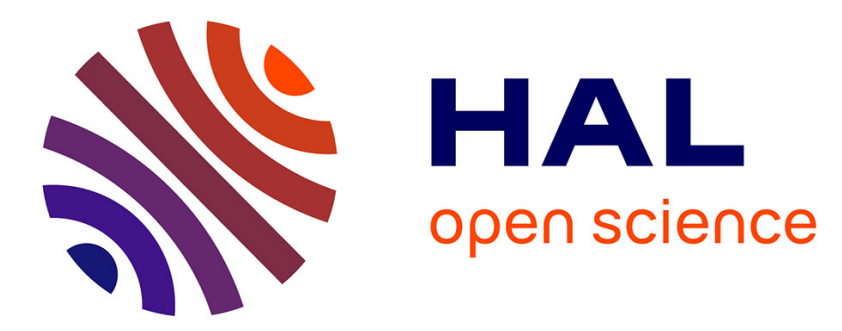

\title{
La binationalité en procès : non-dits et hypocrisies d'un débat récurrent
}

\author{
Vincent Geisser
}

\section{To cite this version:}

Vincent Geisser. La binationalité en procès : non-dits et hypocrisies d'un débat récurrent. Migrations Société, 2011, 136 (4), pp.3. 10.3917/migra.136.0003 . halshs-02113021

\section{HAL Id: halshs-02113021 https://shs.hal.science/halshs-02113021}

Submitted on 30 Apr 2019

HAL is a multi-disciplinary open access archive for the deposit and dissemination of scientific research documents, whether they are published or not. The documents may come from teaching and research institutions in France or abroad, or from public or private research centers.
L'archive ouverte pluridisciplinaire HAL, est destinée au dépôt et à la diffusion de documents scientifiques de niveau recherche, publiés ou non, émanant des établissements d'enseignement et de recherche français ou étrangers, des laboratoires publics ou privés. 


\section{LA BINATIONALITÉ EN PROCÈS : NON-DITS ET HYPOCRISIES D’UN DÉBAT RÉCURRENT \\ Vincent Geisser}

Centre d'information et d'études sur les migrations internationales | «Migrations Société »

$2011 / 4 \mathrm{~N}^{\circ} 136$ | pages 3 à 12

ISSN 0995-7367

Article disponible en ligne à l'adresse :

https://www.cairn.info/revue-migrations-societe-2011-4-page-3.htm

Distribution électronique Cairn.info pour Centre d'information et d'études sur les migrations internationales.

(C) Centre d'information et d'études sur les migrations internationales. Tous droits réservés pour tous pays.

La reproduction ou représentation de cet article, notamment par photocopie, n'est autorisée que dans les limites des conditions générales d'utilisation du site ou, le cas échéant, des conditions générales de la licence souscrite par votre établissement. Toute autre reproduction ou représentation, en tout ou partie, sous quelque forme et de quelque manière que ce soit, est interdite sauf accord préalable et écrit de l'éditeur, en dehors des cas prévus par la législation en vigueur en France. Il est précisé que son stockage dans une base de données est également interdit. 


\section{LA BINATIONALITÉ EN PROCÈS : NON-DITS ET HYPOCRISIES D'UN DÉBAT RÉCURRENT}

Vincent GEISSER

«Le droit n'est pas le moyen le plus approprié pour régler les difficultés résultant de la plurinationalité, car si le droit peut dicter les comportements, il ne dicte pas les sentiments » ${ }^{1}$.

T 'affaire des quotas de "binationaux" suggérés par certains cadres de la Fédération française de football ${ }^{2}$ n'aura finalement surpris que ceux qui croyaient encore en une certaine "pureté" du champ sportif, censé être à l'abri des vents du racisme et des discriminations. Il est vrai que, depuis 1998 - année de l'inoubliable victoire de l'équipe de France à la Coupe du monde - d'aucuns parmi les journalistes, les politiques, mais aussi les sociologues chantaient en chœur l'exceptionnelle tolérance du milieu sportif hexagonal, présenté comme un "modèle d'intégration" dans une France en panne d'imagination ${ }^{3}$.

Le champ sportif était ainsi érigé en véritable laboratoire sociétal de l'intégration "à la française" capable d'insuffler aux autres secteurs de la société un nouveau souffle régénérateur, où la République allait pouvoir enfin se réconcilier avec elle-même. Le sport en général et le football en particulier devinrent ainsi les symboles d'une France « ouverte à la diversité », censés montrer l'exemple et servir de sources

1. JULIEN-LAFERRIĖRE, François, "La Convention européenne sur la réduction des cas de pluralité de nationalités et sur les obligations militaires en cas de pluralité de nationalités", Migrations Société, vol. 14, n 80, mars-avril 2002, pp. 37-50 (cf. p. 47).

2. Cf. ARFI, Fabrice ; FOUTEAU, Carine ; MATHIEU, Mathilde ; HAJDDENBERG, Michaël, "La binationalité : vrai phénomène, faux problème", Mediapart, 30 avril 2011, http://www.mediapart.fr/ journal/france/300411/la-binationalite-vrai-phenomene-faux-probleme

3. Pour une approche critique du mythe de l'intégration par le sport, voir GASTAUT, Yvan, Le métissage par le foot. L'intégration mais jusqu'où ?, Paris : Éd. Autrement, 2008, 184 p. 
d'inspiration à la promotion d'un modèle de bonne gouvernance ${ }^{4}$, conciliant le dynamisme néolibéral et le multiculturalisme sociétal. Il s'en est fallu de peu pour que la formule faussement généreuse «Black-Blanc-Beur » devienne la nouvelle devise de la République médiatique. Alors que, précisément, elle constituait déjà, selon nous, le symptôme patent d'une forme d'ethnicisation larvée de notre société qui n'est pas sans relation avec la "tension discriminatoire" que connaît aujourd'hui le champ sportif. L'apologie des "minorités visibles" sur les terrains de football contient en creux la stigmatisation des "minorités nuisibles", tant les mythes politiques sont éminemment ambivalents et réversibles, comme nous l'ont appris les spécialistes de l'imaginaire national $1^{5}$.

i l'univers footballistique hexagonal n'est sans doute pas davantage "immunisé" contre les tendances ethnicisantes et racialisantes que les autres champs sociaux, il convient toutefois d'éviter d'en faire l'incarnation d'une certaine "beaufitude franchouillarde", sous-entendant par là que l'origine majoritairement "populaire" de ses membres les prédestinerait naturellement à l'intolérance. En somme, selon cette vision réductrice, le football serait le sport le plus exposé au racisme, car le "plus populaire" et le "moins intellectuel" des sports français. Du coup, il ne serait pas étonnant que les cadres $\mathrm{du}$ football, dont l'idéologie serait réputée plus proche du "populisme" que du "multiculturalisme", s'en prennent à la binationalité de certains de leurs joueurs.

On ne reviendra pas ici sur les limites de ce type d'analyses, qui connait (malheureusement) une certaine légitimation scientifique et qui vise à attribuer le succès actuel des thèses d'extrême droite à la déshérence politique des milieux populaires, victimes de la crise ${ }^{6}$. Car, en réalité, les polémiques autour de la binationalité ou de la plurinationalité de certains de nos concitoyens ne constituent pas un

4. La diversité comme "méthode" de marketing donne lieu à la rédaction de nombreux manuels. Voir entre autres BARTH, Isabelle ; FALCOZ, Christophe (sous la direction de), Le management de la diversité. Enjeux, fondements et pratiques, Paris : Éd. L'Harmattan, 2007, 312 p.

5. Cf. GIRARDET, Raoul, Mythes et mythologies politiques, Paris : Éd. du Seuil, 1990, 210 p.

6. Cf. COLLOVALD, Annie, Le "populisme du FN", un dangereux contresens, Bellecombe-enBauges : Éd. du Croquant, 2004, 253 p. 
"pur produit" de l'imaginaire xénophobe des acteurs sportifs. Comme le rappelle fort pertinemment l'historien Patrick Weil, c'est une polémique déjà ancienne et qui fait l'objet depuis de très nombreuses années d'une instrumentalisation politico-électorale : «Bien avant que le Front national ne s'y oppose, la binationalité a été contestée par l'extrême droite, dès le début du siècle dernier, et a notamment mobilisé l'Action française pendant la III République. Leur argument était que ces binationaux étaient supposément des traîtres au service d'un autre pays. Pour l'Action française, les "métèques" étaient, avec les juifs, les protestants et les francs-maçons, une des quatre puissances confédérées, soupçonnées de vouloir détruire le pays $\gg^{7}$.

En ce sens, les politiques ont devancé les sportifs. Et la controverse secouant le milieu du football français autour de la supposée "binationalité" de certains joueurs n'apparaît que comme le prolongement des débats anxiogènes présents dans le champ politique et les milieux élitaires français. En effet, la suspicion de "double allégeance" est un registre récurrent du débat politique hexagonal, brandi régulièrement, certes, par l'extrême droite, mais relayé par des composantes plus "modérées" appartenant à la droite conservatrice, voire à une certaine gauche nationaliste ${ }^{8}$.

Il est vrai que Marine Le Pen, en cela digne héritière de la ligne politique tracée par son père, a relancé la polémique, en adressant, le 30 mai 20II, une lettre aux 577 députés français, afin de les mettre en garde contre les dangers de la binationalité ${ }^{9}$, présentée comme l'un des principaux fléaux de notre société : «Vous le savez, l'État et la nation sont en France indissociables. C'est en effet du sentiment d'appartenance à une même nation que l'État tient dans notre pays sa légitimité. Lorsque

7. WEIL, Patrick, "La binationalité a été contestée par l'extrême droite dès le début du siècle dernier", entretien dans LeMonde.fr, 3 juin 2011, http://www.lemonde.fr/politique/article/2011/06/03/labinationalite-a-ete-contestee-par-l-extreme-droite-des-le-debut-du-siecle_1531406_823448.html

8. Cf. NOIRIEL, Gérard, Racisme : la responsabilité des élites, Paris : Éd. Textuel, 2007, 111 p. ; TÉVANIAN, Pierre, La mécanique raciste, Paris : Éd. Dilecta, 2008, 126 p.

9. Au sens strictement juridique, « il y a plurinationalité quand [...] plusieurs États reconnaissent une même personne comme leur ressortissant. La forme la plus fréquente est la binationalité ou appartenance à la nationalité de deux États. C'est, par exemple, le cas de la personne dont les deux parents sont de nationalité différente et peuvent, en application du jus sanguinis, transmettre l'un et l'autre leur nationalité à leurs enfants. En général, la plurinationalité n'est pas expressément prévue par la législation sur la nationalité, mais résulte de l'absence de disposition la prohibant ". JULIEN-LAFERRIÈRE, François, "La Convention européenne sur la réduction des cas de pluralité de nationalités et sur les obligations militaires en cas de pluralité de nationalité", art. cité, p. 38. 
la solidarité nationale est atteinte, c'est l'autorité de l'État qui se délite. La multiplicité des appartenances à d'autres nations contribue aujourd'hui, et d'une manière de plus en plus préoccupante, à affaiblir chez nos compatriotes l'acceptation d'une communauté de destin, et par là-même à miner les fondements de l'action de l'État. L'explosion du chiffre des binationaux pose aujourd'hui des problèmes dont les Français sont de plus en plus conscients et que le législateur ne peut plus ignorer $\gg^{10}$. La nouvelle présidente du Front national en conclut ainsi à «l'échec patent de la double nationalité ${ }^{11}$.

Fantasme xénophobe circonscrit à une minorité nostalgique d'extrême droite, se réappropriant la thématique conspirationniste des «Quatre États confédérés » de Charles Maurras ? ${ }^{12}$ Au regard des débats politiques actuels, le nationalisme intégral ne semble pas seulement inspirer le Front national, mais également de nombreuses personnalités de l'Union pour un mouvement populaire (UMP), le parti présidentiel, qui ont très largement surenchéri sur les « effets destructeurs » de la binationalité, au premier rang desquels Claude Goasguen, rapporteur de la Mission parlementaire sur le droit de la nationalité qui, dans un pré-rapport publié le 2 I juin $2011^{13}$, préconisait clairement la limitation des cas de double nationalité.

Dans une rhétorique en apparence plus "modérée", la Droite populaire, composante de l'UMP, reprend l'argumentation traditionnelle de l'extrême droite contre la plurinationalité, la rendant responsable du délitement de l'identité nationale. Plus encore, elle entend proposer une réforme profonde de la nationalité française dans un sens "nationaliste radical", tout en se revendiquant de l'antiracisme, attestant de cette capacité à manipuler des valeurs contradictoires, ce qui renvoie à ce fameux «humanisme sécuritaire ${ }^{14}$ que nous pointions du doigt au fil de nos éditoriaux : « Notre pays est confronté à une montée du racisme.

10. LE PEN, Marine, "Lettre aux députés français", 30 mai 2011, http://www.francesoir.fr/sites/default/ files/imagesweb/lettre_lepen.pdf.

11. Ibidem.

12. BIRNBAUM, Pierre, Un mythe politique: la "République juive": de Léon Blum à Mendès France, Paris : Éd. Fayard, 1988, $417 \mathrm{p}$.

13. Pour avoir accès au pré-rapport, voir 38248 le-rapport-de-la-mission-parlementaire-sur-lanationalite[1].pdf. Voir aussi NUNES, Éric, "L'UMP Claude Goasguen attaque la double nationalité avant de reculer", LeMonde.fr, 22 juin 2011, http://www.lemonde.fr/politique/article/2011/ 06/22//-ump-claude-goasguen-attaque-la-double-nationalite-avant-de-reculer_1539204_823448.html

14. GEISSER, Vincent, "Des 'banlieues de l'Europe' aux banlieues de l'Hexagone : le triomphe de la doctrine de 'l'humanisme sécuritaire'” (éditorial), Migrations Société, vol. 17, n 102, novembredécembre 2005, pp. 3-11. 
Dans ce contexte malsain, la France doit s'emparer de cette question de la binationalité. On ne peut pas vouloir réussir l'intégration en l'écartant de la réflexion. Il est certes impossible, comme le suggèrent certains extrémistes, de supprimer la binationalité, mais il faut la limiter. Je souhaite que l'on commence par enregistrer les situations de double nationalité au moment des actes de mariage, de naissance ou de naturalisation. On disposerait ainsi à terme d'un registre des binationaux. En France aujourd'hui, on ne sait pas combien ils sont, sans doute 4 à 5 millions. Je souhaite aussi qu'on aille progressivement vers une limitation de la double nationalité par le biais de discussions bilatérales avec les pays ${ }^{15}$.

En somme, si l'on suit la logique argumentative de Claude Goasguen et de ses acolytes de la Droite populaire (Lionnel Luca, Jean-Paul Garraud, Philippe Meunier, Christian Vanneste, etc.), s'il existe aujourd'hui du racisme dans la société française, c'est la faute des plurinationaux qui, à force de jouer avec leurs multiples identités nationales, finissent par susciter un effet de boomerang négatif dans la population majoritaire, à savoir ces Français "de souche" qui se sentent légitimement menacés dans leur identité : CQFD ! En deux mots, le racisme en France, c'est la faute des binationaux et des plurinationaux!

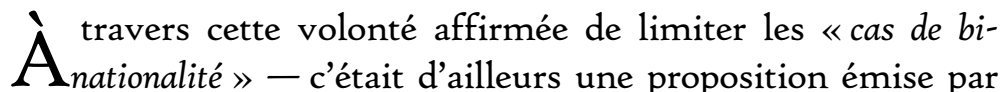
Malek Boutih en 2005 dans un rapport confidentiel pour le Parti socialiste ${ }^{16}$ - on pourrait y voir une certaine forme de "sincérité patriotique" visant à revivifier la citoyenneté en diminuant les "zones grises" du point de vue de la législation française et du droit international. En résumé, ces "réformateurs" de la nationalité chercheraient par tous les moyens à redynamiser l'implication des citoyens dans le "projet national" et donner davantage consistance à la démo-

15. Interview de GOASGUEN, Claude, par GORCE, Bernard, "Nous allons vers une réaffirmation de la nationalité", La Croix du 8-5-2011, http://www.la-croix.com/Actualite/S-informer/France/ Claude-Goasguen-Nous-allons-vers-une-reaffirmation-de-la-nationalite-_EG_-2011-05-08-613675

16. Rappel de la proposition développée par Malek Boutih en 2005 : «Les différentes conventions bilatérales signées avec les pays tiers devront être renégociées afin de clarifier les statuts civils ou de nationalité des citoyens originaires de ces pays. L'objectif est de mettre fin aux statuts binationaux ainsi qu'aux pratiques non conformes au code civil français, en matière de mariage et de filiation particulièrement", cité par GEISSER, Vincent, "Malek Le Pen ou Jean-Marie Boutih ?", oumma.com, 17 mai 2005, http://oumma.com/Malek-Le-Pen-ou-Jean-Marie-Boutih 
cratie française. Mais derrière cette rhétorique bien huilée aux accents "très républicains", l'on décèle une part de non-dit qui révèle en creux une stigmatisation à géométrie variable de la binationalité.

À lire les déclarations des détracteurs de la binationalité, l'on peut légitimement se poser la question : leurs discours visent-ils indistinctement tous les binationaux? Au fil des argumentaires, l'on devine aisément que c'est principalement, pour ne pas dire exclusivement, la binationalité des Français issus de l'immigration postcoloniale - les Noirs et les Arabes pour faire simple - qui pose problème, comme le rappelle la politologue Séverine Labat : «Peu chaut, en effet, à nos dirigeants que des Français possèdent la nationalité norvégienne, comme c'est le cas d'Eva Joly, candidate potentielle à l'élection présidentielle de 2012, et qui, si elle venait à être élue, disposerait du feu nucléaire. Mais l'admettrait-on d'un Franco-Algérien? Le doute est permis, car c'est bien la binationalité d'anciens ressortissants de l'empire qui alimente un imaginaire dont on peut douter qu'il soit lui-même décolonisé $\gg{ }^{17}$.

Sans verser nécessairement dans l'obsession postcoloniale qui consisterait à expliquer tous les phénomènes discriminatoires actuels par un héritage colonial mal digéré ${ }^{18}$, l'on ne peut toutefois qu'être frappé par le fait que la binationalité de certains citoyens français paraît plus dérangeante et plus illégitime que pour d'autres, révélant une véritable hypocrisie, voire un double langage, chez ses pourfendeurs, comme le souligne avec ironie le journaliste franco-algérien Akram Belkaïd : «Il serait intéressant de savoir si ce que prône Goasguen concerne les autres binationaux, notamment les Franco-Américains, les Franco-Allemands sans oublier les Franco-Israéliens. Seront-ils tous concernés par le fameux registre? Devront-ils renoncer à leur autre citoyenneté sous peine de perdre des droits, politiques ou autres, en France? Ou alors, va-t-on nous expliquer qu'il existe des binationaux plus français que d'autres? Qu'il existe des binationaux potentiellement plus déloyaux vis-à-vis de la France que d'autres? Et si ce débat sur les binationaux ne revenait pas à remettre au

17. LABAT, Séverine, "Binationalité : notre futur", Le Monde du 11-6-2011, http://www.lemonde.fr/ idees/article/2011/06/11/binationalite-notre-futur_1534992_3232.html ; LABAT, Séverine, La France réinventée : les nouveaux bi-nationaux Franco-Algériens, Paris : Éd. Publisud, 2010, 272 p.

18. Nous partageons en cela une partie des critiques formulées par Guy Pervillé sur la tendance à tout ramener au facteur postcolonial : PERVILLÉ, Guy, "Réponse à l'article de Séverine Labat", Blog personnel de Guy Pervillé, 14 juin 2011, http://guy.perville.free.fr/spip/article.php3?id_article=262 
goût du jour les propos pestilentiels sur les "vrais Français" et les autres » ${ }^{19}$. Car, à travers les débats récurrents sur la binationalité, c'est bien la suspicion de "double allégeance" qui transparaît, comme si ces «mauvais Français » ${ }^{20}$ (les descendants de l'immigration maghrébine, africaine et turque), par défaut d'intégration sociale, culturelle ou religieuse, étaient susceptibles de trahir les valeurs fondamentales de la nation. Au-delà des apparences de la rigueur des argumentaires juridiques avancés par les prétendus "réformateurs" de la nationalité, la polémique sur la binationalité n'est donc que le réceptacle de fantasmes anxiogènes et de crispations identitaires sur le supposé délitement de notre identité nationale confrontée à la "mondialisation" et à l'“immigration", thèmes privilégiés du parti lepéniste.

Dourtant, l'ambivalence des positionnements autour de la thématique de la binationalité et/ou de la plurinationalité ne se limite ni à la situation présente ni au contexte franco-français. Dans une analyse fort détaillée, François Julien-Laferrière a bien montré comment certains textes européens contribuaient à véhiculer une vision relativement "frileuse" et "dramatisante" à l'excès de la binationalité, présentée comme un facteur de perturbation de la citoyenneté. Ainsi, par exemple, la Convention européenne de 1963 « repose sur la considération, énoncée dans son bref préambule, "que le cumul de nationalités est une source de difficultés". Elle en déduit qu'il convient "de réduire autant que possible, dans les relations entre États membres, les cas de pluralité de nationalités", ce qu'elle déclare "répond[re] au but poursuivi par le Conseil de l'Europe". Cette triple affirmation est contestable $\gg^{21}$. Et l'auteur de conclure, à l'opposé du registre anxiogène de la Convention européenne de 1963, que «la plurinationalité paraît être une richesse, voire une nécessité pour l'individu : une richesse, en ce qu'elle est le signe, la traduction en termes juridiques de l'appartenance à

19. BELKAïD, Akram, "La binationalité au banc des accusés", SlateAfrique, 17 mai 2011, http://www.slateafrique.com/2063/en-france-la-binationalite-au-banc-des-accuses

20. FASSIN, Didier, "Nous sommes tous de mauvais Français", Télérama, n 3133, 27 janvier 2010.

21. JULIEN-LAFERRIĖRE, François, "La Convention européenne sur la réduction des cas de pluralité de nationalités et sur les obligations militaires en cas de pluralité de nationalités", art. cité, p. 39. 
plusieurs collectivités humaines, à plusieurs cultures qui ne sont pas forcément contradictoires mais, le plus souvent, complémentaires ${ }^{22}$.

La suspicion à l'égard des binationaux n'est pas seulement instrumentalisée politiquement de ce côté-ci de la Méditerranée, mais aussi très largement dans les pays d'origine (Maghreb, pays de l'Afrique noire et Turquie). Les autorités consulaires de ces Etats ont constamment combattu la citoyenneté française de "leurs" ressortissants, assimilée à une forme de trahison identitaire et de reniement patriotique. Le contexte d'effervescence nationaliste au moment des indépendances se traduisait directement dans le droit : «Les codes de la nationalité ont compté parmi les premiers textes de loi adoptés par les États maghrébins à leur indépendance. Ils ont été entérinés en 1954 en Libye, 1956 en Tunisie, 1958 au Maroc, 1961 en Mauritanie et 1963 en Algérie. Les conditions d'accès à la nationalité répondaient à la nature de ces États à l'époque. Essentiellement fondées sur le droit du sang, elles permettaient une transmission inégalitaire du lien national, la femme n'étant pas considérée comme un vecteur de continuité identitaire. Limité à sa portion congrue, le droit du sol ne fondait un accès à la communauté que sous condition d'allégeance $\gg^{23}$.

Plus récemment, on se souvient de la déclaration de feu le roi du Maroc, Hassan II, qui sur une chaîne du service public (Antenne 2, ancêtre de France 2), avait clairement exprimé son opposition à l'intégration des résidents marocains à l'étranger (RME) et son hostilité farouche à l'idée qu'ils puissent posséder la nationalité d'un pays européen : «Je suis contre l'intégration. Le bon immigré est celui qui acquiert une formation, une valeur ajoutée, et qui rentre chez lui $\gg^{24}$.

Le royaume du Maroc ne fut pas le seul à dénoncer la binationalité: des États comme l'Algérie et la Tunisie ont longtemps pratiqué une forme de "chantage au passeport", interdisant jusqu'à une période récente aux Franco-Maghrébins de rentrer sur leur territoire avec des papiers français. Au-delà des discours officiels, les «Z'immigrés 》 et leurs enfants faisaient l'objet d'une suspicion généralisée de la part

22. Ibidem, p. 46.

23. PERRIN, Delphine, "Identité et transmission du lien national au Maghreb : étude comparée des codes de la nationalité", L'Année du Maghreb, III | 2007, mis en ligne le $1^{\text {er }}$ novembre 2010, consulté le 26 juin 2011, http://anneemaghreb.revues.org/394

24. HASSAN II, interviewé par DUHAMEL, Alain, émission L'heure de vérité, Antenne 2, 17 décembre 1989. 
de sociétés maghrébines qui comprenaient mal leur nationalité française, vue comme une forme de reniement national et, pire, religieux : devenir français était non seulement identifié à un acte antipatriotique, mais aussi à une forme d'apostasie. Aussi le FrancoMaghrébin se voyait-il traité en double apostat, à l'égard de sa nation (l'Algérie, le Maroc et la Tunisie, le Mali, le Burkina Faso, la Mauritanie, etc.) et à l'égard de sa religion (l'islam). Il est vrai que depuis quelques années les positions des autorités officielles et des acteurs sociaux ordinaires se sont quelque peu assouplies ${ }^{25}$.

Si la binationalité n'est toujours pas reconnue officiellement par les États maghrébins, elle est souvent tolérée et est davantage comprise par les populations des pays d'origine qui finissent par saisir les "avantages existentiels" de leurs émigrés à posséder plusieurs nationalités. Du côté des binationaux eux-mêmes, les tabous tendent à être surmontés. Si dans les décennies précédentes il était encore difficile de s'affirmer Français vis-à-vis de ses "cousins" restés dans le pays d'origine, aujourd'hui le sentiment de "honte" ou d'indignité lié à la double nationalité semble appartenir au passé, comme nous l'analysions déjà dans l'une de nos recherches réalisée à la fin des années 1990: «Contrairement aux précédentes décennies (1970-1980), il n'existe plus vraiment de tabou à se revendiquer simultanément français et algérien (ou marocain ou tunisien), même si les individus savent au fond d'eux-mêmes que leur vie est en France et qu'ils ne retourneront jamais dans leur pays d'origine. Brandir le drapeau national algérien, marocain ou tunisien pendant la Coupe du Monde de football et soutenir passionnément l'équipe de France, c'est finalement faire mentir par ses pratiques sociales les discours normatifs sur l'intégration à la française, tels qu'ils s'expriment dans le champ politique, voire dans certains écrits sociologiques ${ }^{26}$. En somme, les pratiques sociales des individus et des groupes viennent démentir toute la charge "dramatique" entourant la binationalité, qui tend de plus en plus à être vécue sur un mode banalisé.

De ce point de vue, les révolutions démocratiques dans le monde arabe ont constitué un extraordinaire laboratoire d'observation de ce

25. Cf. PERRIN, Delphine, "Identité et transmission du lien national au Maghreb", art. cité.

26. GEISSER, Vincent ; KELFAOUI, Schérazade, "La nation d'origine réinventée. La persistance du 'mythe national' chez les Français originaires du Maghreb ", Confluences Méditerranée, $\mathrm{n}^{\circ} 39$, automne 2001, pp. 27-35 (voir pp. 33-34). 
processus paradoxal de "banalisation/valorisation" de la binationalité, nous permettant de dépasser les préjugés anxiogènes. D'un côté, les Français d'origine arabo-berbère n'ont eu aucun mal à s'identifier aux revendications démocratiques de leurs "concitoyens" des pays d'origine, contribuant à les relayer dans les sociétés européennes sous forme de mobilisations et d'actions de solidarité en faveur des insurgés et des familles victimes de la répression. Au lendemain du départ des dictateurs Ben Ali et Moubarak, ces binationaux francotunisiens ou franco-égyptiens ont même revendiqué une participation active sur les scènes politiques des États d'origine, y compris en tant qu'électeurs, candidats et élus des futurs Parlements démocratiques. Sur ce plan, l'on peut dire qu'ils ont cherché à se fondre dans les enjeux des pays d'origine, en se faisant utiles, tout en restant “discrets" pour ne pas gêner les acteurs “autochtones". D'un autre côté, en tant que Français binationaux, ils ont fait valoir une certaine spécificité, jouant très largement sur leur francité pour affirmer à la fois un capital de compétences citoyennes et une expérience démocratique, censés éclairer et accompagner les transitions politiques en cours dans le monde arabe.

La binationalité au service de l'universalisme démocratique : c'est peutêtre l'une des leçons qu'il convient de tirer des révolutions arabes vécues de ce côté-ci de la Méditerranée ${ }^{27}$ !

Marseille, le 26 juin 2011

27. Exemple parlant, Kamel Jendoubi, président du Réseau euro-méditerranéen des droits de l'homme (REMDH) et acteur majeur du mouvement associatif franco-maghrébin en région parisienne, a été élu en mai 2011 président de la nouvelle Instance supérieure indépendante pour les élections (ISIE) en Tunisie chargée de superviser le processus électoral de l'Assemblée constituante de l'après-Ben Ali. On pourrait évoquer de nombreux autres cas de Franco-Maghrébins jouant un rôle sur les scènes politiques arabes en transition tout en assumant pleinement leur francité. 